\title{
Beating the memory drum: Rapid sequential visual presentation (RSVP) on a $4 \mathrm{~K} \mathrm{PDP}-8$
}

\author{
IRA FISCHLER \\ University of Florida, Gaine sville, Florida $\$ 2611$
}

\begin{abstract}
A program for presenting alphanumeric strings in rapid succession on a videoterminal is described. Rates of up to 30 items/sec can be obtained with relatively "slow" systems by creating a push-up stack in the terminal's display memory and scrolling the items past a small display window during presentation. Advantages of such a program are discussed, and the system is compared to mechanical techniques of rapid sequential visual presentaiion.
\end{abstract}

The use of computing systems in psychological research is often more than a matter of convenience; there is a large class of problems which is difficult, if not impossible, to investigate without the kind of control offered by computers. One example is situations where complex response-contingent events are to be presented (e.g., Maitland, 1970). Another type of experiment for which on-line systems are particularly well suited involves the visual presentation of a large number of alphanumeric stimuli in sequence at very short interitem intervals. Research employing this technique includes studies of target detection in visual search (e.g., Fischler, 1975), sentence perception (e.g., Forster, 1970) and short-term memory (e.g., Pfafflin, 1974), among others.

The simple "memory drum" is adequate for listlearning experiments, where the number of items presented is small and the interstimulus intervals (ISIs) are in the range of 1 to several seconds. As the analysis of perceptual events becomes increasingly molecular, however, presentation rates from 10 to $50 \mathrm{items} / \mathrm{sec}$ have become desirable. In target-detection tasks, for example, performance can remain above chance with presentation rates of upwards of $40 \mathrm{items} / \mathrm{sec}$ in some cases (Sasaki, Note 1). Commercially available light tachistoscopes (e.g., the Iconix system) can easily handle such rates, but only for three or four stimuli on a given trial. There have been a number of special-purpose systems designed with as many as 10 channels (e.g., Eriksen \& Spencer, 1968). The failure of such systems to be adopted generally is due to the rapid increase in the complexity of the logic and structure of the system, and in the difficulty of running successive trials smoothly, as the number of channels increases.

Several other mechanical systems have been implemented for studies involving rapid sequential visual presentation (or RSVP; Forster, 1970). One of these (Lawrence \& Sasaki, 1970) uses a stroboscopic light to "stop action" on a sheet of paper moving steadily past a viewing window. Rates of up to 40 items/sec can be obtained, but the lamps are prone to failure at such speeds, and are costly to replace. The interfacing of the solid state logic used to drive the lamp and the relays needed to operate the movement of the stimulus list has also been problematic. Other drawbacks include a good deal of eyestrain for the subject (who in fact has the strobe light flashing in his face, tempered only by the stimulus sheet) and a great deal of work for the experimenter, who must type a separate list for each presentation order desired, randomize the lists for each student, and change lists in the machine for each trial. A second mechanical approach has been to use motion pictures, photographing each stimulus in turn for a given list, and then presenting the film at various speeds (e.g., Forster, 1970). Here the construction of the stimuli is even more time-consuming than with typewritten lists; and, again, a separate strip is required for each list.

A number of investigators have resorted to computerdriven CRTs to perform RSVP experiments, including Pfafflin (1974), Sperling, Budiansky, Spivak, and Johnson (1971), and Stemberg and Scarborough (Note 2). The present paper describes a program for presenting a sequence of alphanumeric stimuli on a videoterminal at rates of up to $50 \mathrm{items} / \mathrm{sec}$, which has been implemented in the Cognition and Perception Laboratory at the University of Florida on a 4K PDP-8/F. The program is written in assembly language (PAL8), but an equivalent program could be handily constructed with a higher level language such as real-time BASIC, if at least $8 \mathrm{~K}$ of core were available. The original hardware configuration included a Teletype for entering the stimuli and obtaining hard copies of any response latencies recorded, and a SuperBee alphanumeric videoterminal with ASCII keyboard for displaying stimuli and obtaining latency data.

\section{PROGRAM DESCRIPTION}

The heart of the program consists of five modular sections. The first two of these are used at the outset of a particular experiment. First, a set of stimuli are placed into core from either the videoterminal keyboard or the tape reader of the Teletype. The second section creates a series of lists, according to whatever constraints the design calls for. The lists are also in core, and consist of pointers to the appropriate stimuli in the specified order. 
The third and fourth sections are used during a given experimental session. The third accepts a rate of presentation from the videoterminal keyboard and then presents to the subject whatever instructions are called for. This rate can be changed at any point during the session. The subject is given a sequence of list numbers and enters these himself to summon successive tria's. This allows the subject to pace himself, and also frees some core for other uses. The fourth section actually presents the stimuli in sequence for a given list, controlled by a crystal clock. In a target-detection condition, one of the list items is displayed before the sequence begins, and reaction time is measured from the presentation of the target during RSVP to the subject's keypress response; the reaction time is then stored in core. The final section of the program converts the reaction times to decimal base as they are output to the Teletype, along with any additional information required concerning each trial. The entire program resides in 3 pages of core, leaving the rest for the stimuli, list pointers, and data collection.

One of the major problems faced in the construction of the program was the relatively slow speed of the interface between the PDP-8/F and the videoterminal. We were limited to a rate of 300 baud, or about $33 \mathrm{msec} /$ character. This was slow enough to make the writing of each item appear to take time to the subject, which was unacceptable. Moreover, the fastest obtainable presentation rate, given an average of 5 characters/item and a 10 -msec pause between successive items, was about 5 items/sec.

Our solution to this problem was to create, in essence, a memory drum in the display memory of the videoterminal. A common feature of CRT terminals is the ability to scroll up or down whatever information is currently being displayed; this requires a two-character command. Using the scrolling function of the terminal, each item of a given list is written, and the display is scrolled up one line, creating what amounts to a "pushup" stack in the terminal. An opaque screen placed over the display prevents the subject from previewing the list. Presentation now proceeds by scrolling the items down past a small window at the desired rate, much as is done with the Lawrence and Sasaki (1970) stroboscopic apparatus. This technique increased the obtainable rate to about 15 items $/ \mathrm{sec}$; more importantly, writing time was now limited only by the speed of the raster scan, which was for our purposes instantaneous. The number of items that could be presented on a given trial was about 250 , since the display memory of the SuperBee terminal was "economical" in suppressing trailing blanks. Another advantage to the scrolling technique is that the cursor, which on most terminals cannot be suppressed, can be neatly tucked away at the bottom of the screen, adding neither visual noise nor extraneous apparent movement to the presentation.

The limitation on baud rate is not atypical for a basic system in a small research program. Use of the
Decwriter LA36 serial interface card would provide 300 baud, for example; and DEC's recently marketed "Classic" system-which, although designed primarily for educational uses, seems attractive enough to find its way into numerous research labs-runs its videoterminal at 600 baud.

\section{CONCLUSION}

The RSVP program has been evolving along with our hardware and software capabilities. Obtaining a highspeed interface (KL8-JA) allowed us to push the scrolling presentation to the limits obtainable with the raster scan, or about 50 items/sec. (The KL8-JA, which is switchable from 110 to 9,600 baud, is now standard on the PDP-8/E and 8/F computer systems.) Addition of a Floppy disk system meant being able to use a larger stimulus set, and store the data for further analysis. Use of a programmable clock has streamlined the program somewhat. Nonetheless, the basic structure of the program has remained unchanged.

The RSVP procedures described here allow discrete instantaneous presentation of stimuli at "perceptual" rates even on relatively "slow" systems with a minimum of memory. The result is an enjoyable (for the subject), flexible, and reliable system which, compared to the mechanical alternatives, makes only small demands on the experimenter's time, either before or during the experimental session.

\section{REFERENCE NOTES}

1. Sasaki. E. H. Influence of the number and type of targets upon rapid scanning of word lists. Unpublished $\mathrm{PhD}$ dissertation. $19^{7} 0$.

2. Sternberg. S.. \& Scarborough, D. L. Parallel testing of stimuli in visual search. Paper presented at the International Symposium on Visual Information Processing and Control of Motor Activity. Bulgaria, July 1969.

\section{REFERENCES}

Eriksen, C. W.. \& Spencer, T. J. Visual search under conditions of very rapid sequential input rates. Perception \& Psychophysics. 1968. 2. 197.202.

FISCHLER, 1. Detection and identitication of words and letters in stimulated visual search of word lists. Memory \& Cognition. $19^{75}, 3,175-182$.

Forster, K. I. Visual perception of rapidly presented word sequences of varying complexity. Perception \& Psychophysics. 1970, 8, 215-221.

LAWRENCE, D. H.. \& SASAKI, E. H. An N-channel stroboscopic tachistoscope for typewritten stimuli. Behavior Research Methods \& Instrumentation. 1970, 2, 63.65.

MaItland. A. The effects of presentation order in multitrial free recall. Cited by R. J. Fitzhugh \& R. Glaser. A generalpurpose computer system for a research and development center. Amercun Psychologist. 1975, 30, 219-225.

Pfaffin. S. M. The total time hypothesis, recall strategies, and memory for rapidly presented word strings. Memory \& Cognition. 1974. 2. 236-240.

Sperling, G., Budiansky, J., Spivak, J. G., \& Johnson, M. C. Extremely rapid visual search: The maximum rate of scanning letters for the presence of a numeral. Science, 1971, 174, $307-311$. 